\title{
Crystalline Dipeptide Nanobelts Based on Solid-Solid Phase Transformation Self-Assembly and Their Polarization Imaging of Cells
}

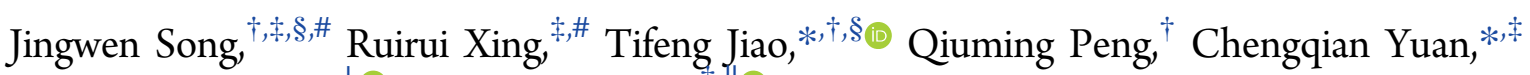 \\ Helmuth Möhwald, ${ }^{\perp}$ and Xuehai Yan $^{*},,_{(\infty)}$
}

\author{
${ }^{\dagger}$ State Key Laboratory of Metastable Materials Science and Technology, Yanshan University, Qinhuangdao 066004, P. R. China \\ ${ }^{\ddagger}$ State Key Laboratory of Biochemical Engineering, Institute of Process Engineering, Chinese Academy of Sciences (CAS), Beijing \\ 100190, P. R. China \\ ${ }^{\S}$ Hebei Key Lab of Applied Chemistry, School of Environmental and Chemical Engineering, Yanshan University, Qinhuangdao \\ 066004, P. R. China \\ "Center for Mesoscience, Institute of Process Engineering, Chinese Academy of Sciences, Beijing 100190, P. R. China \\ ${ }^{\perp}$ Max Planck Institute of Colloids and Interfaces, Am Mühlenberg 1, 14476 Potsdam/Golm, Germany
}

Supporting Information

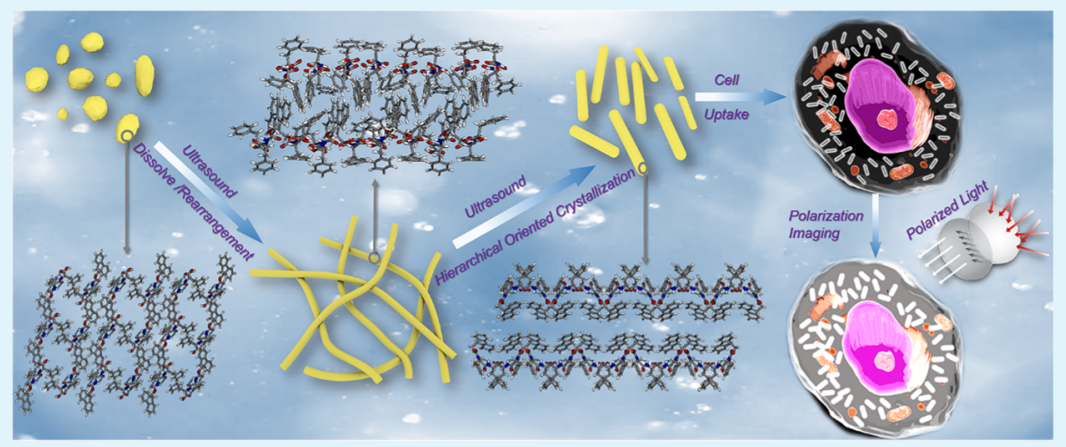

ABSTRACT: Controlled phase transformation involving biomolecular organization to generate dynamic biomimetic selfassembly systems and functional materials is currently an appealing topic of research on molecular materials. Herein, we achieve by ultrasonic irradiation the direct solid-solid transition of bioinspired dipeptide organization from triclinic structured aggregates to nanofibers and eventually to monoclinic nanobelts with strong polarized luminescence. It is suggested that the locally high temperature and pressure produced by cavitation effects cleaves the hydrophobic, $\pi-\pi$ stacking or self-locked intramolecular interactions involved in one phase state and then rearranges the molecular packing to form another well-ordered aromatic dipeptide crystalline structure. Such a sonication-modulated solid-solid phase transition evolution is governed by distinct molecular interactions at different stages of structural organization. The resulting crystalline nanobelts are for the first time applied for polarization imaging of cells, which can be advantageous to directly inspect the uptake and fate of nanoscale delivery platforms without labeling of fluorescent dyes. This finding provides a new perspective to comprehend the dynamic evolution of biomolecular self-organization with energy supply by an external field and open up a facile and versatile approach of using anisotropic nanostructures for polarization imaging of cells and even live organisms in future.

KEYWORDS: dipeptide, nanobelt, phase transformation, self-assembly, polarization imaging

\section{INTRODUCTION}

Self-assembly, widely existing in nature at all scales, plays crucial roles in preparation of functional architectures and in elucidating the complex life evolution process. ${ }^{1,2}$ Peptide selfassembly provides an effective approach to mimic and understand living systems because of their good biocompatibility, responsiveness to external stimuli, and versatile structural programmability. ${ }^{3-11}$ One key feature of real-life systems is that they are often not in thermodynamic equilibrium but in kinetically trapped metastable states determined by the self- assembly pathway, which significantly enriches the complexity and functionality. ${ }^{12,13}$ The primary reason is that molecular selfassembly is the result of synergism of multiple noncovalent interactions between building blocks, which are reversible and dynamic in nature. ${ }^{14}$ Therefore, increasing attention has been paid to phase transformations involved in artificial biomimetic

Received: November 24, 2017

Accepted: December 29, 2017

Published: December 29, 2017 
Scheme 1. Schematic Illustration of the Crystalline Dipeptide Nanobelt Formation under Ultrasonic Irradiation and Their Application for the Polarization Imaging of Cells



self-assembly systems. ${ }^{15-18}$ Sol, gel, and crystal are the phases most studied in the field of peptide self-assembly. In general, phase behavior can be modulated by external stimuli such as solvent, $\mathrm{pH}$, temperature, and ion strength. ${ }^{3,19-21}$ In the past decades, many efforts have been devoted to phase transitions focusing on sol-gel, gel-crystal, gel-sol, and even sol-gelcrystal transformations confirmed by morphology changes and diffraction. ${ }^{17,22-26}$ Recently, the solid-solid phase transformation has come up as a promising area because not only is it more effective for the controlled self-assembly with desired functions but also it provides insight into the dynamic evolution process in life evolution. ${ }^{27,28}$ For example, Li et al. achieved the tunable phase transformation from self-assembled organogel to a well-defined crystal utilizing cryogenic treatment. ${ }^{29}$ However, the realization and underlying mechanism of a solid-solid transition is usually difficult because of the high local energy minimum in a crystalline phase connected with little mobility. Thus, extreme conditions (high or low temperature/pressure) are required for these transitions to occur, and high-resolution measurements are needed to capture intermediate states of the transformations. Therefore, it is mandatory to explore an effective strategy and simple yet comprehensive model systems to achieve such a phase transformation and unravel the underlying molecular mechanisms.

Ultrasound has been used as an effective tool to fabricate hydrogels of biomolecules and induce the gel-gel transition because ultrasonic irradiation can break intramolecular interactions and locally high temperatures allow molecular rearrangement into stronger intermolecular interactions. ${ }^{30-33}$ Suitable conformation changes may then play a crucial role in directing the molecular self-assembly behavior. It is hypothesized that a sample in one well-organized state can be converted into other newly ordered or disordered architectures taking advantage of the extreme local temperature and pressure generated by ultrasonic cavitation. These conditions may exist only over nanoseconds and may move the system out of a metastable state to convert it into another metastable or stable state.

Among the various biomolecules, short aromatic peptides are popular as a type of self-assembly building block because of the functions of bioactive peptides combining aromaticity and hydrophobicity of aromatic groups. ${ }^{34}$ One outstanding case is the amyloid-derived diphenylalanine peptide modified with $\mathrm{N}$ fluorenylmethoxycarbonyl (Fmoc-FF), which has been reported to self-assemble into well-defined nanofibrous structures through a multiscale hierarchical assembly process driven by various intermolecular interactions including hydrophobic interaction, hydrogen bonding, and $\pi-\pi$ stacking. ${ }^{35}$ Herein, we directly observed the morphological and structural transformation of dipeptide self-assembly over time under ultrasound irradiation and ultimately obtained crystalline nanobelts with narrow dispersion, which are $\sim 1.5 \mu \mathrm{m}$ in length and $\sim 250$ $\mathrm{nm}$ in width. Moreover, the well-organized nanobelts exhibit decent polarization imaging capability in cells (Scheme 1). This finding gives insight into the understanding of the dynamics of the self-assembly evolution process and promises more applications of bioderived anisotropic nanostructures in biomedical applications such as polarization imaging of cells and even live organisms in future.

\section{EXPERIMENTAL SECTION}

2.1. Materials. N-Fluorenylmethoxycarbonyl diphenylalanine (Fmoc-FF) was laboratory reagent grade purchased from Bachem Company. Hydrochloric acid $(\mathrm{HCl})$ is a product of Beijing Chemical Works. All other reagents were purchased from Sigma-Aldrich. Water was prepared in a double-stage Millipore Milli-Q Plus purification system.

2.2. Preparation of Crystalline Dipeptide Nanobelts. Briefly, the Fmoc-FF nanobelts are fabricated by sonicating the dipeptide powder (Fmoc-FF, $2 \mathrm{mg}$ ) in Tris- $\mathrm{HCl}(\mathrm{pH}=7.5,1 \mathrm{~mL}$ ) for various exposure times (30 s, 1, 3, 5, and $10 \mathrm{~min})$. Ultrasonic treatment was conducted by a Kunshan KQ5200DE ultrasonic instrument with the powder kept at $100 \mathrm{~W}$.

2.3. Characterization. Scanning electron microscopy (SEM) images were taken with a Hitachi S-4800 SEM with samples placed on the silicon slices. A thin layer of Pt was sputtered to increase the conductivity of the samples. Transmission electron microscopy (TEM) images were recorded by using a JEM-1011 TEM with samples placed onto the carbon-coated copper grids. Atomic force microscopy (AFM) images were collected on a Bruker (USA) Dimension ion operated in tapping mode with a ScanAsyst-Air cantilever under ambient conditions. X-ray diffraction (XRD) data were collected at room temperature on a Empyrean (PANalytical, Nederland) instrument equipped with a $\mathrm{Cu}$ filter with $\mathrm{Cu} \mathrm{K} \alpha_{1}$ radiation $(\lambda=1.5406 \AA)$. All the assemblies during the Fmoc-FF structural evolution at $2 \mathrm{mg} / \mathrm{mL}$ were spread on glass slides as films and allowed to dry at air prior to data collection. Thermogravimetric analysis (TGA) was conducted on Diamond TG-DTA6300 from room temperature to $300{ }^{\circ} \mathrm{C}$ at a constant rate of $20 \mathrm{~K} / \mathrm{min}$ in a nitrogen atmosphere. Different assemblies were dried under vacuum until their weights were constant prior to the TGA measurements. Crosspolarized microscopy images were acquired on an Olympus polarized microscope system BX53. The circular dichroism (CD) spectra in the range of 190-300 nm were collected by a Chirascan spectrometer at room temperature. Fourier transform infrared (FTIR) spectra were 

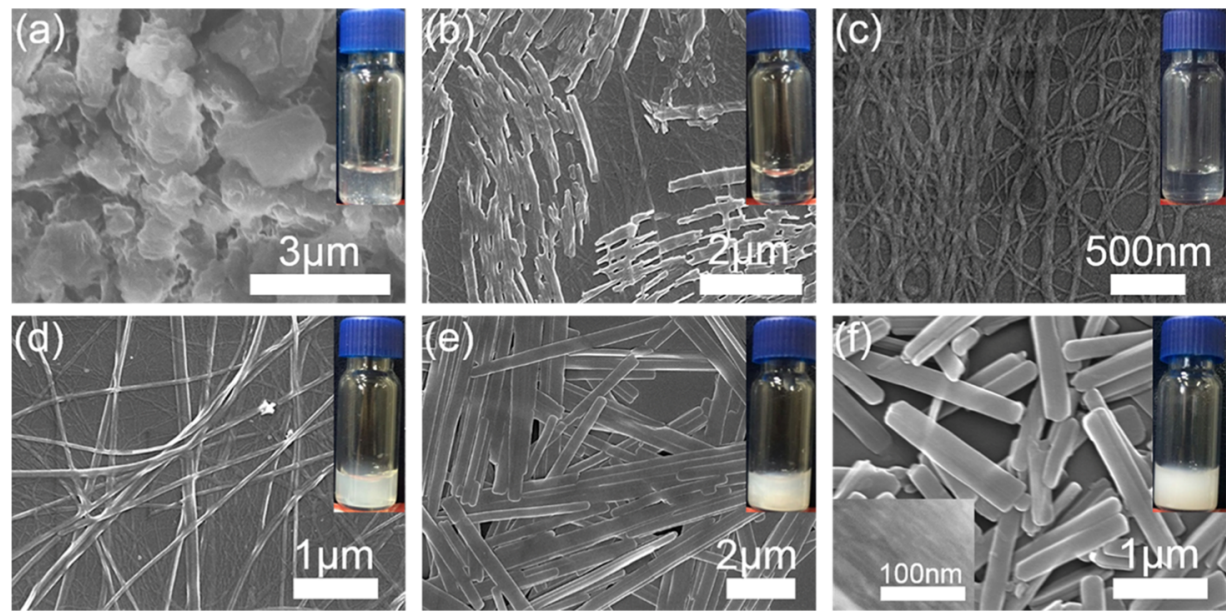

Figure 1. SEM images of nanobelt formation of the Fmoc-FF in Tris- $\mathrm{HCl}(\mathrm{pH}=7.5)$ solution at different sonication time lengths: (a) $0 \mathrm{~s}$, (b) $30 \mathrm{~s}$, (c) $1 \mathrm{~min},(\mathrm{~d}) 3 \mathrm{~min},(\mathrm{e}) 5 \mathrm{~min}$, and (f) $10 \mathrm{~min}$. The green arrows denote the hierarchically oriented organization of the nanobelts.
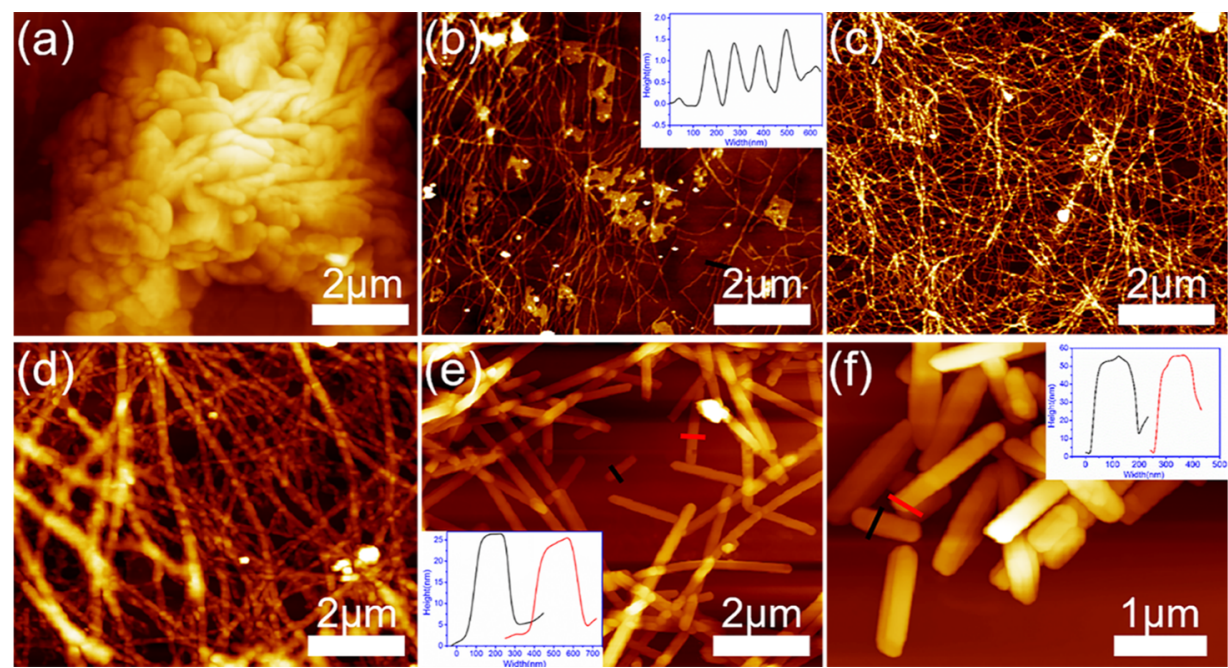

Figure 2. AFM images of nanobelt formation of the Fmoc-FF in Tris- $\mathrm{HCl}(\mathrm{pH}=7.5)$ solution at different sonication time lengths: (a) $0 \mathrm{~s}$, (b) $30 \mathrm{~s}$, (c) $1 \mathrm{~min},(\mathrm{~d}) 3 \mathrm{~min},(\mathrm{e}) 5 \mathrm{~min}$, and (f) $10 \mathrm{~min}$. Inset pictures in (b,e,f) exhibit the height profiles along black lines or red lines.

recorded by a TENSOR-27 infrared spectrometer. The hydrogels were vacuum-dried before mixing with $\mathrm{KBr}$ powder. The fluorescence (FL) spectra and ultraviolet-visible absorption spectroscopy were acquired using model FL-4500 and UV-2600 spectrometers.

2.4. Cell Imaging Experiments. MCF-7 cells were cultured in RPMI 1640 medium consisting of $10 \%$ fetal bovine serum, $100 \mathrm{U} / \mathrm{mL}$ penicillin, and $100 \mu \mathrm{g} / \mathrm{mL}$ streptomycin. Cells were incubated with $0.05 \mathrm{mg} / \mathrm{mL}$ of Fmoc-FF nanobelts at $37{ }^{\circ} \mathrm{C}$ for $24 \mathrm{~h}$. After washing with phosphate buffer saline (PBS) three times to remove the excessively remaining Fmoc-FF nanobelts, the cells were incubated with a freshly prepared culture medium at room temperature. Polarized light images of the mounted cells were obtained through an Olympus polarized microscope system BX53.

2.5. Rietveld Refinement for the XRD Patterns. The optimized geometry of Fmoc-FF with minimized energy is obtained at the level of $\omega \mathrm{B} 97-\mathrm{XD} / 6-311$. The harmonic vibrational frequency calculations on the optimized geometries were also performed to ensure the structures at local minima. The optimized geometry of Fmoc-FF with minimized energy is applied to the refinement of crystalline structures in association with powder XRD data. Pawley refinement and Rietveld refinement were performed to optimize the lattice parameters. The molecular organization in the lattice is achieved by using the simulated annealing method in the Reflex Powder Solve module and further improved by Rietveld refinement in Materials Studio 8.0.

\section{RESULTS AND DISCUSSION}

The dipeptide nanobelts were prepared by ultrasound treatment of Fmoc-FF powder suspended in Tris- $\mathrm{HCl}$ of $\mathrm{pH}=7.5$. SEM and AFM images depict the formation process in detail. It is evident that, for the sample without sonication treatment, a large number of aggregates consisting of nanosheets were observed (Figures 1a and 2a). In sharp contrast, such aggregates gradually disappeared upon sonication, and nanofibers with lengths of several tens of micrometers were generated (Figure 1b). Furthermore, the 3D AFM height image clearly displays the spatial dimensions of the nanofibers with average thickness of $\sim 1.2 \mathrm{~nm}$ (Figure $2 \mathrm{~b}$ ). A pronounced change of the dispersion from turbid to clear indicates an instantaneous dissolution-reassembly process. With sonication time extended to $1 \mathrm{~min}$, entangled nanofibrous networks consisting of interconnected nanofibers were observed, which are usually responsible for gelation (Figures $1 \mathrm{c}$ and 2c). Moreover, the diameters of the nanofibers range from 20 to $100 \mathrm{~nm}$, revealing the existence of nanofiber bundles. Further increasing the sonication time to $3 \mathrm{~min}$, such nanofiber bundles further grew and small amounts of nanoribbons with more than tens of micrometers in length and $\sim 100 \mathrm{~nm}$ in width emerged, 


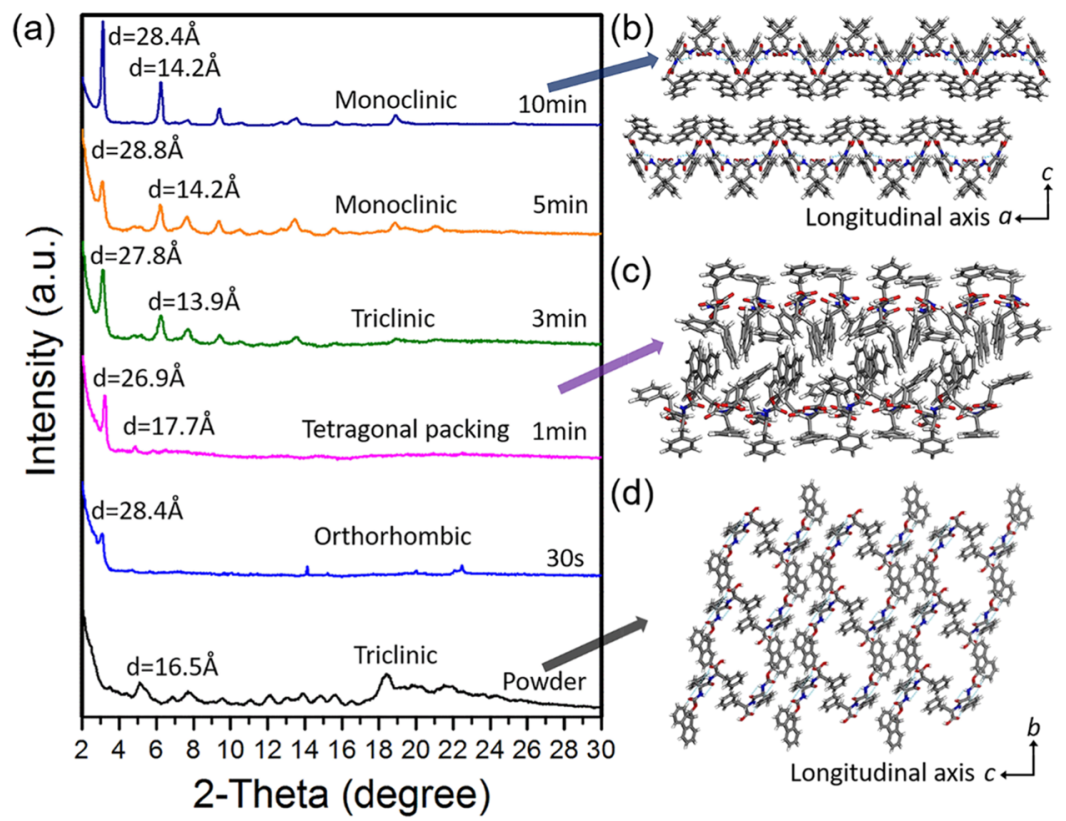

Figure 3. Time-dependent XRD patterns of different assemblies during the ultrasound-induced structural evolution of the Fmoc-FF in Tris- $\mathrm{HCl}$ (a). The molecular packing patterns of the nanobelts (b), nanofibers (c), and starting powder (d).

along with a significant change of the solution from transparent to opaque again (Figures $1 \mathrm{~d}$ and $2 \mathrm{~d}$ ). Upon ultrasound exposure during $5 \mathrm{~min}$, substantial nanobelts that are over several micrometers in length and $\sim 20 \mathrm{~nm}$ in thickness emerged under persistent ultrasonic irradiation (Figures le and 2e). Finally, well-defined short nanobelts with lengths of about $1.5 \mu \mathrm{m}$ and widths on the order of $250 \mathrm{~nm}$ were obtained (Figure 1f). Insights into the surface of such nanobelts demonstrate the stacking patterns of thin nanobelts (Figure 2f), which reveals their hierarchically oriented organization. TEM images further display the structural evolution from irregular aggregates to entangled nanofiber bundles to thin nanoribbons and eventually to narrowly dispersed nanobelts with small lengths and aspect ratios (Figure S1 in Supporting Information). These observations agree well with the timedependent SEM and AFM images. All morphology results reveal that well-defined Fmoc-FF nanobelts could be achieved in a hierarchically oriented organization manner with the assistance of sonication.

XRD measurements were performed to gain insight into the formation process of dipeptide nanobelts assisted by ultrasound irradiation. As shown in Figure 3a, Fmoc-FF powder exhibits a series of weak peaks, and the indexing of these peaks yields a triclinic packing mode in the powder. The distance corresponding to the peak at $2 \theta=5.22^{\circ}$ is $16.5 \AA$, which is ascribed to the molecular length of Fmoc-FF monomer (as shown in Figure S2a in Supporting Information). At the initial stage of sonication treatment $(30 \mathrm{~s})$, the peak at $5.22^{\circ}$ disappeared, demonstrating a gradual dissolution process of the above sample. A new peak at $3.08^{\circ}$ emerged, indicating the assemblies' molecular organization changes leading to the lattice changes from triclinic to orthorhombic upon sonication treatment. Further extending the ultrasonic duration to $1 \mathrm{~min}$, two diffraction peaks remained in the small angle range, indicating the ordered organization of the entangled nanofibers. Such nanofibers in a quickly formed kinetically favorable metastable state can supposedly overcome a barrier for molecular rearrangement to achieve a thermodynamically more stable crystal state with assistance of local and short time energy accumulation provided by ultrasonic irradiation. By prolonging the sonication to $3 \mathrm{~min}$, several new peaks appeared, and the distance ratio of the second diffraction peak to the primary one is $1: 2$, which reveals the formation of lamellar organization. The distance of $27.8 \AA$ is close to the combined length of two Fmoc-FF molecules (Figure S2b) through stacking the Fmoc moiety, suggesting the occurrence of bilayer or interdigitated structures. Moreover, the indexing of the XRD patterns of samples submitted to $3 \mathrm{~min}$ sonication reveals the recurrence of a triclinic lattice. For assemblies obtained after 5 and $10 \mathrm{~min}$ sonication treatments, typical lamellar structures also emerged, the indexing of the diffraction pattern confirmed the formation of crystalline Fmoc-FF with a monoclinic lattice. Overall, the time-dependent XRD pattern reveals that Fmoc-FF samples underwent a dynamic solid-solid transformation from kinetically controlled metastable states to a thermodynamically favorable state.

Matrix-assisted laser desorption/ionization time-of-flight mass spectrometry was carried out to probe the chemical composition, in special to answer the question if sonication changed the molecule. As shown in the mass spectrum of the assembled nanobelts (Figure S3 in Supporting Information), two characteristic peaks at $\mathrm{m} / z 557$ and 573 corresponding to $[\mathrm{M}+\mathrm{Na}]^{+}$and $[\mathrm{M}+\mathrm{K}]^{+}$of the Fmoc-FF molecule reveal the chemical structure of the building blocks. To elucidate the molecular arrangement of assemblies with different sonication time lengths, Pawley and Rietveld refinements were performed to optimize the lattice parameters of the finally formed nanobelts and of the starting powder. The structural parameters are provided in Table S1 in Supporting Information and the most possible molecular organizations are shown in Figure $3 \mathrm{~b}$,d. The monoclinic lattice of nanobelts consists of four Fmoc-FF molecules and is stabilized by offsetting $\pi-\pi$ stacking along with $\mathrm{O}-\mathrm{H} \cdots \mathrm{O}, \mathrm{N}-\mathrm{H} \cdots \mathrm{N}$ hydrogen bonds as shown in Figure $3 \mathrm{~b}$. With respect to the molecular organization of the starting powder (Figure 3d), the triclinic lattice comprises two Fmoc-FF molecules held together by T-shaped $\pi-\pi$ stacking 

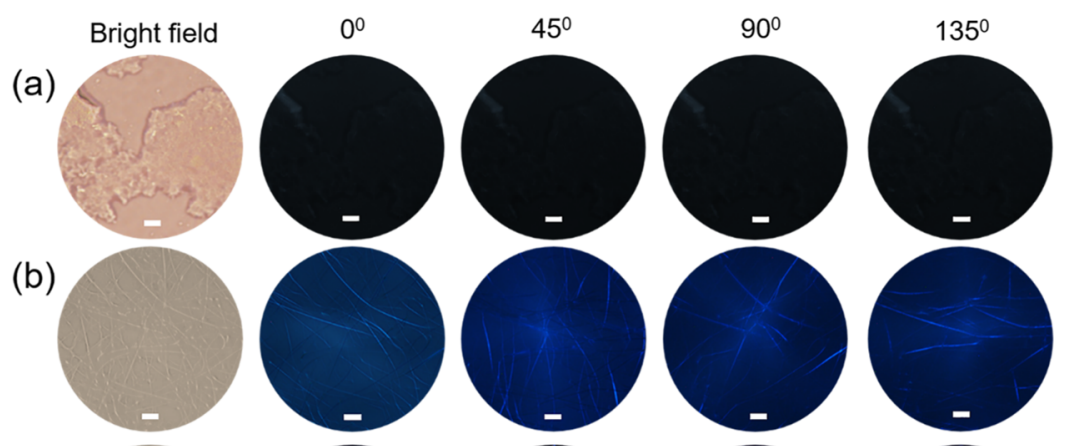

(c)
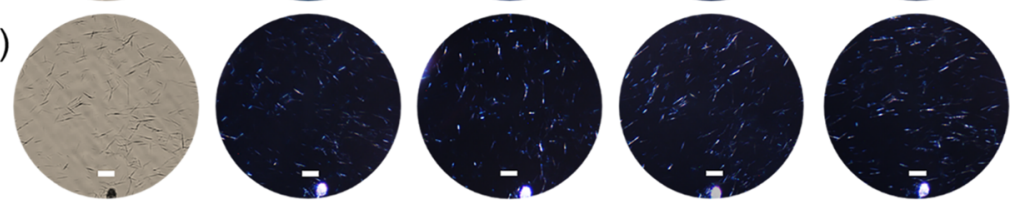

(d)
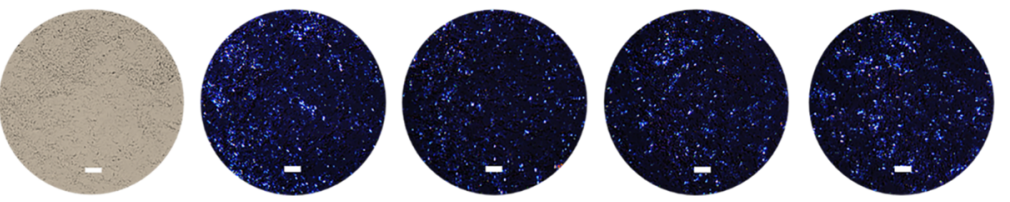

Figure 4. Cross-polarized microscopy images of Fmoc-FF assemblies obtained at different sonication time lengths: (a) 1, (b) 3, (c) 5, and (d) 10 min, showing the kinetic evolution of crystalline nanobelts starting from aggregates spanning the long fibers and belts. All the scale bars: $20 \mu \mathrm{m}$.
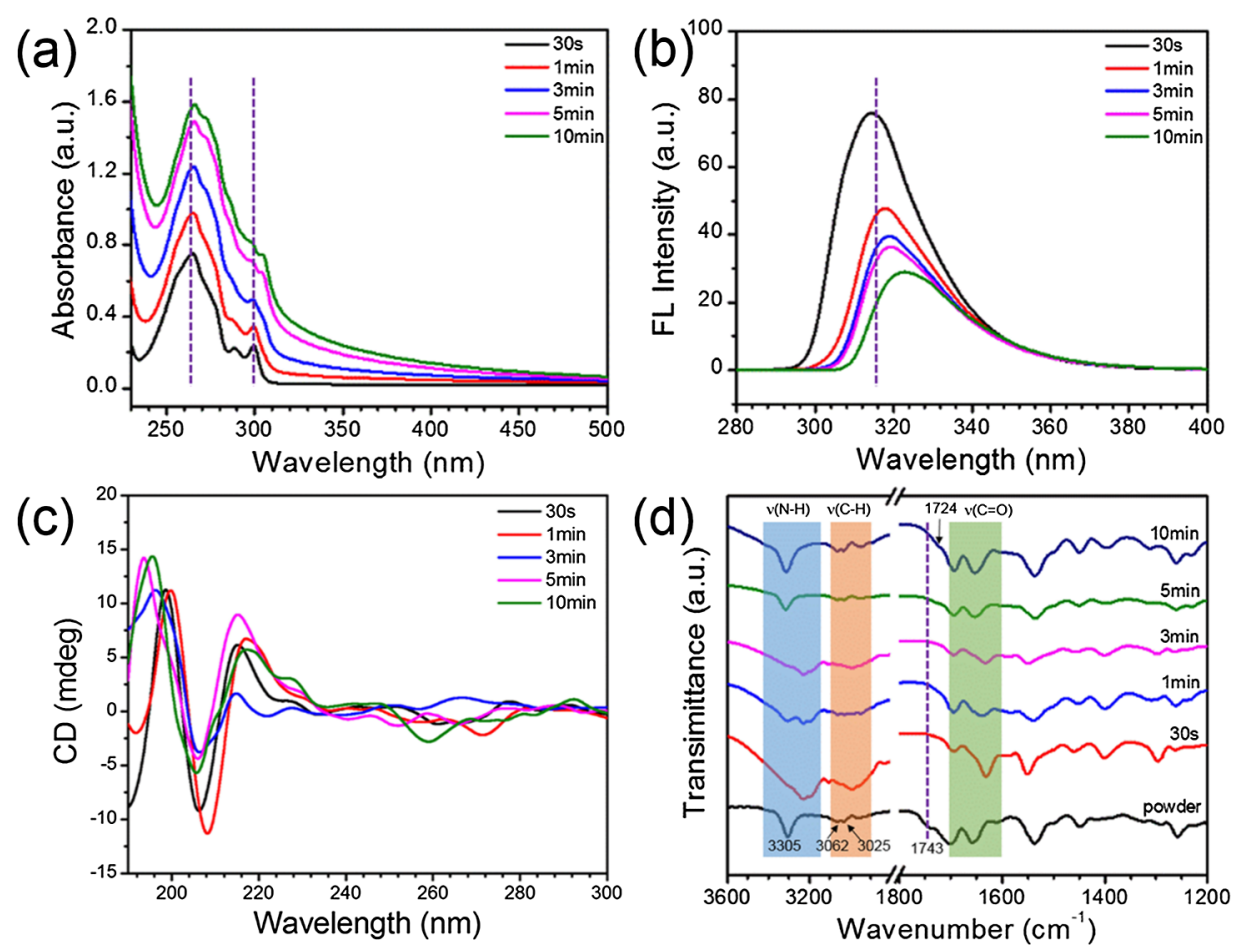

Figure 5. Time-dependent characterization of the ultrasound-induced nanobelt formation process of the Fmoc-FF in Tris- $\mathrm{HCl}(\mathrm{pH}=7.5)$. (a) UVvis spectra, (b) FL spectra, (c) FTIR spectra, and (d) CD spectra.

and stabilized by intermolecular hydrogen bonds. Combining with the XRD pattern of interconnected nanofibers and the molecular organization in Fmoc-FF hydrogel proposed by Ulijn et al., ${ }^{24}$ the molecular arrangement in Figure $3 \mathrm{c}$ corresponding to the Fmoc-FF nanofibers is organized in an antiparallel $\beta$ sheet manner, where the Fmoc moieties of alternate $\beta$-sheets are interlocked to generate $\pi$-stacked pairs. Furthermore, we explore the role of water during the self-assembly transformation process of Fmoc-FF under the ultrasound irradiation. It is found that all the ultrasound-induced assemblies exhibit pronounced weight-loss steps in their TGA curves around 100 ${ }^{\circ} \mathrm{C}$, which is ascribed to the removal of water molecules embedded in the lattices (Figure S4 in Supporting Information), indicating their vital role in the peptide selfassembly process, which is consistent with previous reports. ${ }^{19,20}$ Therefore, the variation of molecular geometries, molecular packing modes, and water content modulated by the sustained ultrasonic stimulus results in the solid-solid phase transformations. 
Cross-polarized optical microscopy images were further used to track the formation process of Fmoc-FF nanobelts. No obvious anisotropic birefringence was observed for the interconnected nanofibers (Figure 4a). In sharp contrast, some of the nanoribbons with a large aspect ratio are bright under crossed polarizers (Figure $4 \mathrm{~b}$ ). When we rotate the relative orientation between the polarizer and sample by $45^{\circ}$, the bright ones become very dark, whereas some original nanobelts turn to be bright. Such optical changes suggest that Fmoc-FF dipeptide molecules are uniaxially oriented within the crystalline nanobelts, thus giving rise to strong optical anisotropy of the nanobelts. Similar periodical birefringence changes appeared for the other nanobelts with different aspect ratios, indicating the emergence of crystalline Fmoc-FF structures (Figure 4c,d).

We further investigate the molecular interaction and driving forces leading to such phase transitions through UV-vis, FL, FTIR, and CD spectra. Figure 5a displays time-dependent UVvis spectra during nanobelt formation. With prolonged sonication time, an obvious red shift and broadening of the characteristic UV-vis absorbance band at approximately 262 $\mathrm{nm}$ is observed, indicating spontaneous aromatic-aromatic interactions induced by sonication. The fluorescence emission peaks shown in Figure $5 \mathrm{~b}$ are ascribed to the Fmoc group. Their fluorescence intensity is diminished and red-shifted with increasing time of ultrasound irradiation, further revealing that the aromatic-aromatic stacking interactions are enhanced with persistent ultrasound irradiation. Such enhanced interactions facilitate the formation of a more ordered supramolecular structure. In addition, the CD spectra shown in Figure 5c reveal that all the assemblies obtained during the structural evolution process display two strong bands, namely a negative band approximately at $205-208 \mathrm{~nm}$ and a positive band around 194-198 nm. The band around 205-208 $\mathrm{nm}$ has been previously observed in cross- $\beta$ assemblies of peptides such as $(\mathrm{FKFE})_{n}$ and Ac-(FKFK) $)_{2}-\mathrm{NH}_{2}$, which is resulted from $\pi-\pi$ stacking of the aromatic side chains or the distortion of the $\beta$ sheets. $^{36,37}$ The CD signals between 260 and $300 \mathrm{~nm}$ are attributed to $\pi-\pi^{*}$ transitions between stacked Fmoc groups with an offset face-to-face manner, which is consistent with the molecular arrangement according to the XRD patterns. ${ }^{38,39}$ Moreover, time-dependent FTIR spectra were employed to explore the change in intermolecular interactions during the transition process (Figure 5d). A comparison between the starting powder and the as-obtained supramolecular assemblies reveals that the $\mathrm{N}-\mathrm{H}$ stretching vibration of Fmoc-FF powder at $3305 \mathrm{~cm}^{-1}$ is significantly shifted to the range of $3193-3315$ $\mathrm{cm}^{-1}$. This change indicates the existence of multiple intermolecular hydrogen bonding interactions between FmocFF molecules during the sonication-modulated phase transformation process. In addition to the $\mathrm{N}-\mathrm{H}$ stretching vibration, the $\mathrm{C}-\mathrm{H}$ stretching vibration region also changes significantly. Specifically, for the assemblies achieved by sonication within 3 min, the profile of the $\mathrm{C}-\mathrm{H}$ stretching region becomes broadened compared to the starting powder, revealing various intermolecular interactions involving the $\mathrm{C}-\mathrm{H}$ groups. It should be noted that the FTIR spectrum of crystallized nanobelts exhibits well-defined $\mathrm{C}-\mathrm{H}$ stretching vibrations, which further demonstrates a highly ordered molecular arrangement in such structures. Furthermore, the $\mathrm{C}=\mathrm{O}$ stretching vibration can be used as an effective marker for hydrogen bonding. ${ }^{40,41}$ Two typical stretching bands appeared at 1743 and $1702 \mathrm{~cm}^{-1}$, which are ascribed to the carbonyl $\mathrm{C}=$
O stretching of the free and intramolecular H-bonded carboxylic acid. The disappearance of the former band and red shifts of the bands after sonication treatment reveal the breakage of weaker intramolecular interactions and formation of stronger intermolecular hydrogen bonding interactions. Therefore, ultrasound not only can destroy weak intra/ intermolecular interactions in a kinetically controlled state but can also facilitate molecular arrangements to form a thermodynamically more favorable state. In addition, the intermolecular laterally bonded and bifurcated carboxyl groups characterized by the band around $1724 \mathrm{~cm}^{-1}$, allowing the hierarchically oriented organization of the nanobelts. In view of these results, we can conclude that offset $\pi-\pi$ stacking along with $\mathrm{O}-\mathrm{H} \cdots \mathrm{O}, \mathrm{N}-\mathrm{H} \cdots \mathrm{N}$ hydrogen bonding interactions dominates the molecular arrangement of crystalline nanobelts.

We have performed additional experiments with variation of ultrasound power, peptide concentration, and the kind of solvents to get better insight into the formation mechanism of nanobelts. First, control experiments were also performed with the ultrasonic power at $200 \mathrm{~W}$, as shown in Figure S5 in Supporting Information. For this case, crystalline nanobelts could also be obtained, but with a small aspect ratio compared to those achieved at $100 \mathrm{~W}$. This may lie in the fact that more bubbles collapse resulting from the increasing ultrasonic power significantly break the hydrogen bonding interaction and promote the hydrophobic and aromatic-aromatic stacking interactions between the thin nanobelts. Next, we investigate the structural evolution based on different peptide concentrations. The results reveal that well-defined nanobelts with increasing lengths and aspect ratios can be prepared, which is due to the accelerated self-assembly and crystallization speed resulting from the increased concentration (Figures S6 and S7 in Supporting Information). In addition to the ultrasonic power and the amounts of dipeptide, the control experiment was also conducted in PBS buffer to explore the effect of the solvent on the self-assembly transformation process. It can be found that the nanobelts with length ranging from 2 to $5 \mu \mathrm{m}$ and width around $200 \mathrm{~nm}$ were the predominant products (Figure S8 in Supporting Information). Such phenomena can be ascribed to the enhanced intermolecular hydrogen bonding interaction between dipeptides with the assistance of PBS buffer. Therefore, tunable dipeptide nanobelts can be realized through modulating the parameters of solid-solid transformation selfassembly.

Based on the above results, it can be concluded that different molecular arrangements govern different stages of the sonication-modulated phase transition. Therefore, we suggest a mechanism of formation of the well-defined crystalline nanobelts. Abundant intramolecular hydrogen bonding interactions dominate the starting powder as shown in Figure $3 \mathrm{~d}$. Once such a powder is subjected to ultrasound irradiation, the locally high temperature and pressure produced by cavitation effects destroys the weak intramolecular interactions, hence leading to the damage of the molecular packing models in powder. On the other hand, the hydrophobic interactions and $\pi-\pi$ stacking are enhanced because of the locally high temperature, which facilitates the quick formation of small nuclei and the further growth into entangled nanofibers. The rapid formation of nanofibers gives rise to a locally high concentration of nanofibers, hence boosting the oriented alignment at the long range and nanofiber bundles because of the competition of translational and rotational entropy in view of Onsager theory. ${ }^{42}$ Further growth of such nanofiber bundles 

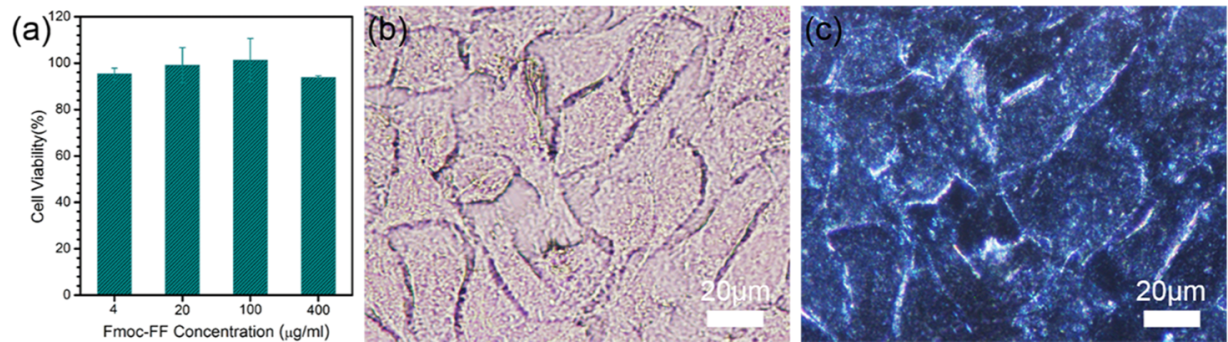

Figure 6. (a) Fmoc-FF concentration-dependent cell viability of MCF-7. Polarization images of MCF-7 cells with uptake of Fmoc-FF nanobelts in (b) bright and (c) cross-polarized fields.

generates thin nanobelts through hierarchically oriented organization. Subsequently, these thin nanobelts would stack laterally through the enhanced hydrophobic interactions triggered by the persistent ultrasonic irradiation. Eventually, crystalline nanobelts formed through the hierarchical crystallization because of the balance between the hydrogen bonding and hydrophobic interactions. Overall, the dissolutionreassembly and the hierarchically oriented crystallization process assisted by the local extreme temperature and pressure produced by ultrasonic cavitation is responsible for the altered molecular geometries and intermolecular interactions, which give rise to the formation of dipeptide crystalline nanobelts.

The high crystalline order gives rise to the emergence of welldefined peptide nanobelts with distinguished polarized luminescence. Compared to traditional bioimaging agents, especially the organic dyes, ${ }^{43,44}$ the crystalline dipeptide nanobelts can significantly extend their potential application in biological fields because of their remarkable advantages. First, such bioderived nanomaterials possess intrinsic biocompatibility and biodegradability, which reduces the side effects at the greatest extent. Second, the crystalline nanobelts with distinguished polarization emission can monitor the intercellular uptake and fate of nanomaterials with no need of any organic dyes, extremely excluding the interference of organic dyes on the surface property of nanomaterials. Last, the nanobelts exhibit long-term photostability/chemical stability and broad polarization excitation spectra yet narrow emission. Therefore, we attempted to take full advantage of the polarized imaging performance in cells. First, we determined the toxicity of the Fmoc-FF nanobelts to the MCF-7 cells by an MTT assay. The dosage-dependent MTT experiments showed that the cellular viabilities are all higher than $95 \%$, even for the highest concentrations, implying that the nanobelts are not toxic and biocompatible to living organisms (Figure 6a). It can be accounted for the flexibility endowed by the laminated organization of the nanobelts, ${ }^{45}$ which makes them deformable when interacting with the cells. Based on this positive result, we conducted the polarized imaging experiments in MCF-7 cells. Excellent polarized light imaging performance and a clear cell profile demonstrate that Fmoc-FF nanobelts can be used as an effective bioimaging agent in living cells and even organisms (Figure 6b). It is the first time to apply well-ordered peptide nanostructures for polarization imaging of cells, which gave them a decisive advantage over inspection of the uptake and fate of nanoscale delivery platforms without labeling by fluorescent dyes.

\section{CONCLUSIONS}

In summary, we have successfully obtained crystalline dipeptide nanobelts based on the solid-solid transformation self- assembly under ultrasonic irradiation. Such a transformation undergoes a kinetic evolution from irregular aggregates to long nanofibers to flexible nanoribbons and eventually to monoclinic nanobelts, where the involved intermediate assemblies enrich the complexity and functionality. This dynamic process can be considered as an intriguing archetypal model to in some sense resemble the complex biomolecular systems. Distinct molecular interactions at different stages dictate the complex structural evolution process including dissolution-reassembly, and the hierarchically oriented crystallization in that ultrasound destroys and homogenizes the intramolecular/intermolecular interactions and reshapes the molecular arrangement to form the thermodynamically favorable peptide crystal with remarkable anisotropy. As a proof-of-concept study, such crystalline nanobelts exhibit distinguished polarization imaging performance of cells. This strategy could be extended to achieve transitions between self-assembly polymorphs in similar aromatic peptides. Moreover, this finding provides more insights into the kinetic evolution process in real biological systems and establishes a noninvasive and cost-effective method to image live organisms based on nontoxic biomolecules.

\section{ASSOCIATED CONTENT}

S Supporting Information

The Supporting Information is available free of charge on the ACS Publications website at DOI: 10.1021/acsami.7b17933.

TEM images of ultrasound-induced nanobelt formation; typical Fmoc-FF monomer and stacked dimer conformation; matrix-assisted laser desorption/ionization time-of-flight mass spectrum of the crystallized nanobelts; TGA curves of starting powder and ultrasoundinduced assemblies; SEM image and XRD pattern of Fmoc-FF nanobelts; SEM images and XRD patterns of ultrasound-induced nanobelt formation at different sonication time lengths; and refined parameters of the starting powder and nanobelts lattices (PDF)

\section{AUTHOR INFORMATION}

\section{Corresponding Authors}

*E-mail: tfjiao@ysu.edu.cn (T.J.).

*E-mail: cqyuan@ipe.ac.cn (C.Y.).

*E-mail: yanxh@ipe.ac.cn. Web: http://www.yan-assembly. org/ (X.Y.).

ORCID $\odot$

Tifeng Jiao: 0000-0003-1238-0277

Helmuth Möhwald: 0000-0001-7833-3786

Xuehai Yan: 0000-0002-0890-0340

Author Contributions

\# J.S. and R.X. contributed equally to this work 


\section{Notes}

The authors declare no competing financial interest.

\section{ACKNOWLEDGMENTS}

We acknowledge financial support from the National Natural Science Foundation of China (project nos. 21473208, 21522307, 21473153, and 91434103), the Talent Fund of the Recruitment Program of Global Youth Experts, the Key Research Program of Frontier Sciences of Chinese Academy of Sciences (CAS, grant no. QYZDB-SSW-JSC034), the CAS President's International Fellowship Initiative (2017DE0004 and 2017VEA0023), as well as the Support Program for the Top Young Talents of Hebei Province, Postdoctoral Science Foundation (no. 2015M580214), and the Scientific and Technological Research and Development Program of Qinhuangdao City (no. 201701B004).

\section{REFERENCES}

(1) Aida, T.; Meijer, E. W.; Stupp, S. I. Functional Supramolecular Polymers. Science 2012, 335, 813-817.

(2) Tao, K.; Makam, P.; Aizen, R.; Gazit, E. Self-assembling peptide semiconductors. Science 2017, 358, No. eaam9756.

(3) Wang, J.; Liu, K.; Xing, R.; Yan, X. Peptide self-assembly: thermodynamics and kinetics. Chem. Soc. Rev. 2016, 45, 5589-5604.

(4) Ariga, K.; Li, J.; Fei, J.; Ji, Q.; Hill, J. P. Nanoarchitectonics for Dynamic Functional Materials from Atomic-/Molecular-Level Manipulation to Macroscopic Action. Adv. Mater. 2016, 28, 1251-1286.

(5) Liu, K.; Xing, R.; Li, Y.; Zou, Q.; Möhwald, H.; Yan, X. Mimicking Primitive Photobacteria: Sustainable Hydrogen Evolution Based on Peptide-Porphyrin Co-Assemblies with a Self-Mineralized Reaction Center. Angew. Chem., Int. Ed. 2016, 55, 12503-12507.

(6) Yan, X. Bio-inspired photosystem for green energy. Green Energy Environ. 2017, 2, 66.

(7) Zou, Q.; Abbas, M.; Zhao, L.; Li, S.; Shen, G.; Yan, X. Biological Photothermal Nanodots Based on Self-Assembly of PeptidePorphyrin Conjugates for Antitumor Therapy. J. Am. Chem. Soc. 2017, 139, 1921-1927.

(8) Liu, K.; Yuan, C.; Zou, Q.; Xie, Z.; Yan, X. Self-Assembled Zinc/ Cystine-Based Chloroplast Mimics Capable of Photoenzymatic Reactions for Sustainable Fuel Synthesis. Angew. Chem., Int. Ed. 2017, 56, 7876-7880.

(9) Cao, M.; Lu, S.; Zhao, W.; Deng, L.; Wang, M.; Wang, J.; Zhou, P.; Wang, D.; Xu, H.; Lu, J. R. Peptide Self-Assembled Nanostructures with Distinct Morphologies and Properties Fabricated by Molecular Design. ACS Appl. Mater. Interfaces 2017, 9, 39174-39184.

(10) Zhan, J.; Cai, Y.; Ji, S.; He, S.; Cao, Y.; Ding, D.; Wang, L.; Yang, Z. Spatiotemporal Control of Supramolecular Self-Assembly and Function. ACS Appl. Mater. Interfaces 2017, 9, 10012-10018.

(11) Xing, R.; Liu, K.; Jiao, T.; Zhang, N.; Ma, K.; Zhang, R.; Zou, Q.; Ma, G.; Yan, X. An Injectable Self-Assembling Collagen-Gold Hybrid Hydrogel for Combinatorial Antitumor Photothermal/Photodynamic Therapy. Adv. Mater. 2016, 28, 3669-3676.

(12) Mattia, E.; Otto, S. Supramolecular systems chemistry. Nat. Nanotechnol. 2015, 10, 111-119.

(13) Yan, X.; Möhwald, H. Organized Peptidic Nanostructures as Functional Materials. Biomacromolecules 2017, 18, 3469-3470.

(14) Wang, J.; Zou, Q.; Yan, X. Peptide Supramolecular SelfAssembly: Structural Precise Regulation and Functionalization. Acta Chim. Sin. 2017, 75, 933-942.

(15) Tantakitti, F.; Boekhoven, J.; Wang, X.; Kazantsev, R. V.; Yu, T.; Li, J.; Zhuang, E.; Zandi, R.; Ortony, J. H.; Newcomb, C. J.; Palmer, L. C.; Shekhawat, G. S.; de la Cruz, M. O.; Schatz, G. C.; Stupp, S. I. Energy landscapes and functions of supramolecular systems. Nat. Mater. 2016, 15, 469-476.

(16) Fukui, T.; Kawai, S.; Fujinuma, S.; Matsushita, Y.; Yasuda, T.; Sakurai, T.; Seki, S.; Takeuchi, M.; Sugiyasu, K. Control over differentiation of a metastable supramolecular assembly in one and two dimensions. Nat. Chem. 2017, 9, 493-499.

(17) Fichman, G.; Guterman, T.; Damron, J.; Adler-Abramovich, L.; Schmidt, J.; Kesselman, E.; Shimon, L. J. W.; Ramamoorthy, A.; Talmon, Y.; Gazit, E. Spontaneous structural transition and crystal formation in minimal supramolecular polymer model. Sci. Adv. 2016, 2, No. e1500827.

(18) Berdugo, C.; Nalluri, S. K. M.; Javid, N.; Escuder, B.; Miravet, J. F.; Ulijn, R. V. Dynamic Peptide Library for the Discovery of Charge Transfer Hydrogels. ACS Appl. Mater. Interfaces 2015, 7, 2594625954.

(19) Wang, J.; Liu, K.; Yan, L.; Wang, A.; Bai, S.; Yan, X. Trace Solvent as a Predominant Factor To Tune Dipeptide Self-Assembly. ACS Nano 2016, 10, 2138-2143.

(20) Wang, J.; Yuan, C.; Han, Y.; Wang, Y.; Liu, X.; Zhang, S.; Yan, $\mathrm{X}$. Trace Water as Prominent Factor to Induce Peptide Self-Assembly: Dynamic Evolution and Governing Interactions in Ionic Liquids. Small 2017, 13, 1702175.

(21) Zhang, R.; Xing, R.; Jiao, T.; Ma, K.; Chen, C.; Ma, G.; Yan, X. Carrier-Free, Chemophotodynamic Dual Nanodrugs via Self-Assembly for Synergistic Antitumor Therapy. ACS Appl. Mater. Interfaces 2016, $8,13262-13269$.

(22) Fichman, G.; Gazit, E. Self-assembly of short peptides to form hydrogels: Design of building blocks, physical properties and technological applications. Acta Biomater. 2014, 10, 1671-1682.

(23) Liyanage, W.; Brennessel, W. W.; Nilsson, B. L. Spontaneous Transition of Self-assembled Hydrogel Fibrils into Crystalline Microtubes Enables a Rational Strategy To Stabilize the Hydrogel State. Langmuir 2015, 31, 9933-9942.

(24) Smith, A. M.; Williams, R. J.; Tang, C.; Coppo, P.; Collins, R. F.; Turner, M. L.; Saiani, A.; Ulijn, R. V. Fmoc-Diphenylalanine Self Assembles to a Hydrogel via a Novel Architecture Based on $\pi-\pi$ Interlocked $\beta$-Sheets. Adv. Mater. 2008, 20, 37-41.

(25) Xing, R.; Li, S.; Zhang, N.; Shen, G.; Möhwald, H.; Yan, X. SelfAssembled Injectable Peptide Hydrogels Capable of Triggering Antitumor Immune Response. Biomacromolecules 2017, 18, 35143523.

(26) Fu, M.; Li, Q.; Sun, B.; Yang, Y.; Dai, L.; Nylander, T.; Li, J. Disassembly of Dipeptide Single Crystals Can Transform the Lipid Membrane into a Network. ACS Nano 2017, 11, 7349-7354.

(27) Duan, Z.; Zhang, Y.; Zhang, B.; Zhu, D. Crystal-to-Crystal Transformation from Antiferromagnetic Chains into a Ferromagnetic Diamondoid Framework. J. Am. Chem. Soc. 2009, 131, 6934-6935.

(28) Kobatake, S.; Takami, S.; Muto, H.; Ishikawa, T.; Irie, M. Rapid and reversible shape changes of molecular crystals on photoirradiation. Nature 2007, 446, 778-781.

(29) Liu, X.; Fei, J.; Wang, A.; Cui, W.; Zhu, P.; Li, J. Transformation of Dipeptide-Based Organogels into Chiral Crystals by Cryogenic Treatment. Angew. Chem. 2017, 129, 2704-2707.

(30) Bardelang, D.; Camerel, F.; Margeson, J. C.; Leek, D. M.; Schmutz, M.; Zaman, M. B.; Yu, K.; Soldatov, D. V.; Ziessel, R.; Ratcliffe, C. I.; Ripmeester, J. A. Unusual Sculpting of Dipeptide Particles by Ultrasound Induces Gelation. J. Am. Chem. Soc. 2008, 130, 3313-3315.

(31) Cravotto, G.; Cintas, P. Molecular self-assembly and patterning induced by sound waves. The case of gelation. Chem. Soc. Rev. 2009, 38, 2684-2697.

(32) Wang, Y.; Zhan, C.; Fu, H.; Li, X.; Sheng, X.; Zhao, Y.; Xiao, D.; Ma, Y.; Ma, J. S.; Yao, J. Switch from Intra- to Intermolecular H-Bonds by Ultrasound: Induced Gelation and Distinct Nanoscale Morphologies. Langmuir 2008, 24, 7635-7638.

(33) Yu, X.; Liu, Q.; Wu, J.; Zhang, M.; Cao, X.; Zhang, S.; Wang, Q.; Chen, L.; Yi, T. Sonication-Triggered Instantaneous Gel-to-Gel Transformation. Chem.-Eur. J. 2010, 16, 9099-9106.

(34) Tao, K.; Levin, A.; Adler-Abramovich, L.; Gazit, E. Fmocmodified amino acids and short peptides: simple bio-inspired building blocks for the fabrication of functional materials. Chem. Soc. Rev. 2016, $45,3935-3953$ 
(35) Yuan, C.; Li, S.; Zou, Q.; Ren, Y.; Yan, X. Multiscale simulations for understanding the evolution and mechanism of hierarchical peptide self-assembly. Phys. Chem. Chem. Phys. 2017, 19, 23614-23631.

(36) Bowerman, C. J.; Liyanage, W.; Federation, A. J.; Nilsson, B. L. Tuning $\beta$-Sheet Peptide Self-Assembly and Hydrogelation Behavior by Modification of Sequence Hydrophobicity and Aromaticity. Biomacromolecules 2011, 12, 2735-2745.

(37) Xing, R.; Yuan, C.; Li, S.; Song, J.; Li, J.; Yan, X. Charge-induced Secondary Structure Transformation of Amyloid-Derived Dipeptide Assemblies from $\beta$-sheet to $\alpha$-helix. Angew. Chem., Int. Ed. 2018, DOI: $10.1002 /$ anie.201710642.

(38) Ryan, D. M.; Anderson, S. B.; Nilsson, B. L. The influence of side-chain halogenation on the self-assembly and hydrogelation of Fmoc-phenylalanine derivatives. Soft Matter 2010, 6, 3220-3231.

(39) Zhu, P.; Yan, X.; Su, Y.; Yang, Y.; Li, J. Solvent-Induced Structural Transition of Self-Assembled Dipeptide: From Organogels to Microcrystals. Chem.-Eur. J. 2010, 16, 3176-3183.

(40) Yan, X.; Su, Y.; Li, J.; Früh, J.; Möhwald, H. Uniaxially Oriented Peptide Crystals for Active Optical Waveguiding. Angew. Chem., Int. Ed. 2011, 50, 11186-11191.

(41) Seo, J.; Hoffmann, W.; Warnke, S.; Huang, X.; Gewinner, S.; Schöllkopf, W.; Bowers, M. T.; von Helden, G.; Pagel, K. An infrared spectroscopy approach to follow $\beta$-sheet formation in peptide amyloid assemblies. Nat. Chem. 2017, 9, 39-44.

(42) Onsager, L. The effects of shape on the interaction of colloidal particles. Ann. N.Y. Acad. Sci. 1949, 51, 627-659.

(43) Fernández-Suárez, M.; Ting, A. Y. Fluorescent probes for superresolution imaging in living cells. Nat. Rev. Mol. Cell Biol. 2008, 9, 929-943.

(44) McVey, B. F. P.; Tilley, R. D. Solution Synthesis, Optical Properties, and Bioimaging Applications of Silicon Nanocrystals. Acc. Chem. Res. 2014, 47, 3045-3051.

(45) Adler-Abramovich, L.; Arnon, Z. A.; Sui, X.; Azuri, I.; Cohen, H.; Hod, O.; Kronik, L.; Shimon, L. J. W.; Wagner, H. D.; Gazit, E. Bioinspired Flexible and Tough Layered Peptide Crystals. Adv. Mater. 2018, DOI: $10.1002 /$ adma.201704551. 